\title{
Lattice Boltzmann simulation of water droplet impacting a hydrophobic plate with a cylindrical pore
}

\author{
Geng Wang, ${ }^{1}$ Linlin Fei, ${ }^{2}$ and Kai H. Luo $\oplus^{1, *}$ \\ ${ }^{1}$ Department of Mechanical Engineering, University College London, Torrington Place, \\ London WC1E 7JE, United Kingdom \\ ${ }^{2}$ Center for Combustion Energy, Department of Energy and Power Engineering, Key Laboratory for Thermal \\ Science and Power Engineering of Ministry of Education, Tsinghua University, Beijing 100084, China
}

(Received 3 March 2020; accepted 8 July 2020; published 7 August 2020)

\begin{abstract}
This work uses a nonorthogonal multiple-relaxation-time lattice Boltzmann (LB) method to simulate a water droplet impacting on a hydrophobic plate with a cylindrical pore. The LB method is based on a pseudopotential multiphase model with tunable contact angles. It is first validated against experimental data and then employed to study the dynamics of a water droplet impacting a perforated hydrophobic plate. A comprehensive parametric study is carried out by varying the pore size, plate thickness, and Weber number. Based on these results, a phase diagram is constructed to describe the different regimes of droplet penetration behaviors after impingement on the perforated plate. Three distinctive regimes are found, that is, continuously dripping, totally crossing, and hanging, and the mechanisms behind them are scrutinized through the analysis of pressure and energy balance. The competition between the dynamic pressure and the capillary pressure determines the formation of the liquid jet below the plate. Viscous dissipation hinders the development of liquid fingering. Based on the balance of the droplet dynamic pressure, capillary pressure, and the viscous pressure losses, the boundary between different regimes is established.
\end{abstract}

DOI: 10.1103/PhysRevFluids.5.083602

\section{INTRODUCTION}

The forced impact of a droplet on a perforated plate is a common phenomenon in technological and medical applications such as inkjet printing, 3D printing, fuel spray, spray cooling of perforated turbine blades, drug production, and cell spheroid culture [1-4]. Using microfluidics, passive and active control of droplet generation can be realized $[5,6]$. The number, size, and speed of droplets passing through a hole have a critical influence on product quality $[2,7,8]$. According to the theories, the inertial effects, capillary effect, and capillary instability govern the droplet impact, permutation, and breakup, respectively [3,9-11]. Especially in the low-Weber-number system, the Laplace pressure and the solid surface wettability represent a significant influence on droplet hydrodynamics $[3,12]$. With the discovery of self-clean and self-propulsion phenomena, the impact dynamics of a droplet on a hydrophobic or hydrophilic surface has become an important research topic [13]. As examples of application, an air-permeable waterproof membrane and an enhancing droplet breakup mesh have already been developed inspired by the related research [14]. Considering the complex physical process behind the phenomenon, a systematic investigation is critical to developing a detailed understanding of droplet-solid surface impact as well as the permeation mechanism when a droplet impacts a pore on a perforated plate.

\footnotetext{
*Corresponding author: k.luo@ucl.ac.uk
} 
There have been some studies on water droplets impacting a holed hydrophobic surface. Both Kumar et al. [15] and Zhang et al. [16] experimentally researched the droplet impact on superhydrophobic meshes. The contact time, maximum spread diameter, and penetration behaviors of the droplet were measured. Lorenceau and Quéré experimentally investigated the impact of a droplet onto a solid plate with a small hole [17]. Based on theoretical analysis, they provided the threshold Weber number for droplets captured by the plate [17]. In the study of Kooij et al. [18], they found that fragmentation after the droplet impacting the mesh is caused by jet instability [18]. Additionally, Delbos et al. [9] studied the influence of wettability of the solid surface when a droplet is forced to impact on the pore. They revealed the mechanisms behind droplet infiltration due to the contribution of the capillary force [9]. Haghani [19] implemented a two-dimensional (2D) lattice Boltzmann (LB) method to simulate the gravity-driven droplet dripping through a hole and proposed four different droplet equilibrium states. They indicated that the droplet impact mechanism needs further investigation, and the simulation domain should be extended to a three-dimensional (3D) configuration to match the realistic situation [19].

The volume of fluid (VOF), level set, and LB method are regarded as three main methods to simulate multiphase flow [20-22]. Compared with the VOF and level set methods, the LB method is especially suited to capturing the phase interface and predicting the interaction between the droplet and the solid surface [21-23], allowing interface deformation, breakup, and merging naturally without additional artificial numerical algorithms. Moreover, the study of Mukherjee et al. [24] showed that the LB method was computationally an order of magnitude more efficient, and the associated spurious velocities at the interfaces were one to three orders of magnitude lower compared with VOF. On the other hand, the basic LB method is incapable of handling a large density ratio (between the liquid phase and vapor phase), and there are severe spurious currents near the phase interface which may reduce the numerical stability and accuracy. In this study, a 3D nonorthogonal multiple-relaxation-time LB model proposed by Fei et al. [25] is applied to enhance the numerical stability. The improved multiphase pseudopotential model proposed by Li et al. [26,27] is employed to achieve a large density ratio, leading to LB simulation of a realistic water droplet impact.

This article aims to investigate the dynamic behaviors of droplet-plate interaction and explore the influence of various geometric and operating parameters. A regime diagram of droplet-plate impact will be constructed, and the mechanisms behind the different regimes will be scrutinized. In the following, Sec. II describes the LB methodology, simulation setup, and model validation. Section III presents the simulation results and discussion, followed by the conclusion of this study in Sec. IV.

\section{METHODOLOGY}

\section{A. The lattice Boltzmann multiphase model}

An efficient nonorthogonal multiple-relaxation-time (MRT) lattice Boltzmann model (LBM) [25] is employed in this study. In the study of Hoang et al. [28], it was shown that the 2D simulation delayed the droplet breakup due to ignoring the Laplace pressure caused by the out-droplet plane. Considering the influence of the 3D capillary effects on the droplet breakup dynamics, we adopt the 3D LB configuration in this study [28,29]. The MRT collision step can be written as [26]

$$
f_{i}^{*}(\boldsymbol{x}, t)=f_{i}(\boldsymbol{x}, t)-\boldsymbol{\Lambda}\left[f_{i}-f_{i}^{\mathrm{eq}}\right]_{(x, t)}+\frac{\Delta t}{2}\left[\bar{F}_{i}(\boldsymbol{x}, t)+\bar{F}_{i}\left(\boldsymbol{x}+\boldsymbol{e}_{i} \Delta t, t+\Delta t\right)\right],
$$

where $f_{i}$ and $f_{i}^{*}$ are discrete distribution functions before and after collision, respectively, $\boldsymbol{x}$ represents the position vector, $t$ is the time, $\bar{F}_{i}$ stands for the forcing terms in the discrete velocity space, and $\boldsymbol{e}_{i}$ are the discrete velocities. The collision operator is $\boldsymbol{\Lambda}=\left(\boldsymbol{M}^{-1} \boldsymbol{S M}\right)$, where $\boldsymbol{M}$ is the transformation matrix, which depends on the raw moment set, and $S$ is a diagonal relaxation matrix. 
The low-Mach equilibrium distribution function $f_{i}^{\text {eq }}$ is given as [25]

$$
f_{i}^{\mathrm{eq}}=\rho \omega\left(\left|\boldsymbol{e}_{i}\right|^{2}\right)\left[1+\frac{\boldsymbol{e}_{i} \cdot \boldsymbol{u}}{c_{s}^{2}}+\frac{\left(\boldsymbol{e}_{i} \cdot \boldsymbol{u}\right)^{2}}{2 c_{s}^{4}}-\frac{(\boldsymbol{u} \cdot \boldsymbol{u})}{2 c_{s}^{2}}\right],
$$

where $\rho$ stands for density, $c_{s}$ is the lattice sound speed and equals $1 / \sqrt{3}$, and $\boldsymbol{u}=\left[u_{x}, u_{y}, u_{z}\right]$ represents the fluid velocity tensor. In the present work, the D3Q19 lattice model is used to construct the lattice Boltzmann model. The weights for the D3Q19 lattice model are $\omega(0)=1 / 3, \omega(1)=$ $1 / 18$, and $\omega(2)=1 / 36$. The discrete velocities $\boldsymbol{e}_{i}=\left[\left|e_{i x},\right| e_{i y}, \mid e_{i z},\right]$ are defined as follows:

$$
\begin{aligned}
\left|e_{i x}\right\rangle= & {[0,1,-1,0,0,0,0,1,-1,1,-1,1,-1,1,-1,0,0,0,0,1,-1,1,} \\
& -1,1,-1,1,-1]^{\mathrm{T}}, \\
\left|e_{i y}\right\rangle= & {[0,0,0,1,-1,0,0,1,1,-1,-1,0,0,0,0,1,-1,1,-1,1,1,-1,} \\
& -1,1,1,-1,-1]^{\mathrm{T}} \\
\left|e_{i z}\right\rangle= & {[0,0,0,0,0,1,-1,0,0,0,0,1,1,-1,-1,1,1,-1,-1,1,1,1,1,} \\
& -1,-1,-1,-1]^{\mathrm{T}},
\end{aligned}
$$

where $i=0,1, \ldots, 18,|\cdot\rangle$ denotes a 19-dimensional column vector, and the superscript $\mathrm{T}$ denotes the transposition. The force terms can be written as [30]

$$
\bar{F}_{i}=\omega\left(\left|\boldsymbol{e}_{i}\right|^{2}\right)\left[\frac{\boldsymbol{e}_{i}-\boldsymbol{u}}{c_{s}^{2}}+\frac{\left(\boldsymbol{e}_{i} \cdot \boldsymbol{u}\right) \boldsymbol{e}_{i}}{c_{s}^{4}}\right] \cdot F,
$$

where $\boldsymbol{F}=\left[F_{x}, F_{y}, F_{z}\right]$ is the total force vector imposed on the fluid. Multiplication of the explicit form of Eq. (1) by the transformation matrix $\boldsymbol{M}$ leads to the collision equation in the moment space as follows [25,31]:

$$
\overline{\boldsymbol{m}}^{*}=\overline{\boldsymbol{m}}-S\left(\overline{\boldsymbol{m}}-\boldsymbol{m}^{\mathbf{e q}}\right)+\left(\boldsymbol{I}-\frac{S}{2}\right) \Delta t \tilde{\boldsymbol{F}}
$$

where $\boldsymbol{I}$ is the unit matrix, the transformation $\overline{f_{i}}=f_{i}-\Delta t \overline{F_{i}} / 2$ has been used to remove the implicit scheme in Eq. (1), $\overline{\boldsymbol{m}}=\boldsymbol{M} \overline{\boldsymbol{f}}, \boldsymbol{m}^{\mathrm{eq}}=\boldsymbol{M} \boldsymbol{f}^{\text {eq }}$, and $\tilde{\boldsymbol{F}}=\boldsymbol{M} \overline{\boldsymbol{F}}$. Following the collision step, the discrete distribution functions are obtained by $\bar{f}^{*}{ }_{i}=\boldsymbol{M}^{-1} \overline{\boldsymbol{m}}^{*}$. By streaming from position $\boldsymbol{x}$ to the neighboring positions $\left(\boldsymbol{x}+\boldsymbol{e}_{i} \Delta t\right)$ along the discrete velocity directions, the updated discrete distribution functions are

$$
\bar{f}_{i}\left(\boldsymbol{x}+\boldsymbol{e}_{i} \Delta t, t+\Delta t\right)=\bar{f}_{i}^{*}(\boldsymbol{x}, t)
$$

The fluid properties in the physical space can be calculated by

$$
\rho=\sum_{i} \bar{f}_{i}, \rho u=\sum_{i} \bar{f}_{i} \boldsymbol{e}_{i}+\frac{\Delta t \boldsymbol{F}}{2} .
$$

In the above formulation, an orthogonal basis vector set has been usually used to construct the transformation matrix $\boldsymbol{M}$, resulting in the classical orthogonal MRT-LBM [32,33]. However, orthogonality is not a necessary condition for stability. In 2014, Lycett-Brown and Luo [34] showed that an MRT-LBM based on a nonorthogonal basis vector set can also enhance the numerical performance compared with the classical single-relaxation-time LBM (SRT-LBM). Recently, Li et al. [35] further showed that a nonorthogonal MRT-LBM can retain the numerical accuracy and simplify the implementation of its orthogonal counterpart. Based on a generalized multiple-relaxation-time scheme [36,37], Fei et al. proposed another nonorthogonal MRT-LBM [25]. Compared with the classical MRT-LBM, the proposed nonorthogonal MRT-LBM simplifies the transformation between the discrete velocity space and the moment space, and exhibits better portability across different discrete velocity models. In this paper, the nonorthogonal MRT-LBM by Fei et al. [25] is employed. 
For the D3Q19 discrete velocity model, the corresponding nonorthogonal transformation matrix $\boldsymbol{M}$ is given as follows:

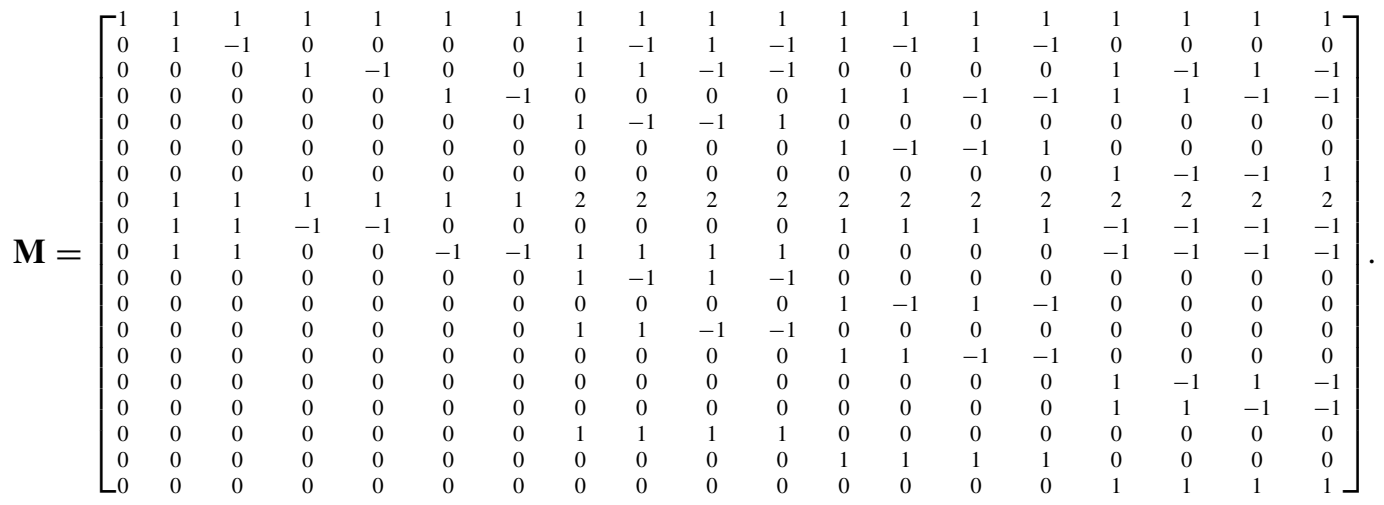

Using the definition $\boldsymbol{m}^{\mathrm{eq}}=\boldsymbol{M} \boldsymbol{f}^{\mathrm{eq}}$, the equilibrium moments can be obtained:

$$
\begin{aligned}
\mathbf{m}^{\mathrm{eq}}= & {\left[\rho, \rho u_{x}, \rho u_{y}, \rho u_{z}, \rho u_{x} u_{y}, \rho u_{x} u_{z}, \rho u_{y} u_{z}, \rho\left(1+\mathbf{u}^{2}\right), \rho\left(u_{x}^{2}-u_{y}^{2}\right),\right.} \\
& \rho\left(u_{x}^{2}-u_{z}^{2}\right), \rho c_{s}^{2} u_{x}, \rho c_{s}^{2} u_{x}, \rho c_{s}^{2} u_{y}, \rho c_{s}^{2} u_{z}, \rho c_{s}^{2} u_{y}, \rho c_{s}^{2} u_{z}, \\
& \left.\rho c_{s}^{2}\left(c_{s}^{2}+u_{x}^{2}+u_{y}^{2}\right), \rho c_{s}^{2}\left(c_{s}^{2}+u_{x}^{2}+u_{z}^{2}\right), \rho c_{s}^{2}\left(c_{s}^{2}+u_{y}^{2}+u_{z}^{2}\right)\right]^{\mathrm{T}} .
\end{aligned}
$$

Similarly, the forcing terms in the moment space are given as

$$
\begin{aligned}
\tilde{\mathbf{F}}= & {\left[0, F_{x}, F_{y}, F_{z}, F_{x} u_{y}+F_{y} u_{x}, F_{x} u_{z}+F_{z} u_{x}, F_{y} u_{z}+F_{z} u_{y}, 2 \mathbf{F} \cdot \mathbf{u},\right.} \\
& 2\left(F_{x} u_{x}-F_{y} u_{y}\right), 2\left(F_{x} u_{x}-F_{z} u_{z}\right), F_{x} c_{s}^{2}, F_{x} c_{s}^{2}, F_{y} c_{s}^{2}, F_{z} c_{s}^{2}, F_{y} c_{s}^{2}, \\
& \left.F_{z} c_{s}^{2}, 2 c_{s}^{2}\left(F_{x} u_{x}+F_{y} u_{y}\right), 2 c_{s}^{2}\left(F_{x} u_{x}+F_{z} u_{z}\right), 2 c_{s}^{2}\left(F_{y} u_{y}+F_{z} u_{z}\right)\right]^{\mathrm{T}} .
\end{aligned}
$$

In addition, the diagonal relaxation matrix $S$ is defined as

$$
\mathbf{S}=\operatorname{diag}\left(s_{0}, s_{1}, s_{1}, s_{1}, s_{2}, s_{2}, s_{2}, s_{2 b}, s_{2}, s_{2}, s_{3}, s_{3}, s_{3}, s_{3}, s_{3}, s_{3}, s_{4}, s_{4}, s_{4}\right) .
$$

The kinematic and bulk viscosities are related to the relaxation parameters for the second-order moments by $v=\left(\frac{1}{S_{2}}-0.5\right) C_{S}^{2} \Delta t$ and $\xi=2 / 3\left(\frac{1}{s_{2 b}}-0.5\right) C_{S}^{2} \Delta t$, respectively. It can be proven through a Chapman-Enskog analysis (see Appendix A in [25]) that such a nonorthogonal MRT-LBM recovers the Navier-Stokes equations in the low-Mach-number limit.

To simulate the multiphase flow, the pseudopotential model is adopted in which the molecular interactions that lead to the phase segregation between phases are represented by a pseudopotential interaction force [38]:

$$
\boldsymbol{F}_{\text {int }}=-G \psi(\boldsymbol{x}) \sum_{i} w\left(\left|\boldsymbol{e}_{i}\right|^{2}\right) \psi\left(\boldsymbol{x}+\boldsymbol{e}_{i} \Delta t\right) \boldsymbol{e}_{i},
$$

where $G$ is the interaction strength which is usually fixed as $-1, \psi$ is the pseudopotential which is dependent on density, and the weights are $w\left(\left|\boldsymbol{e}_{i}\right|^{2}\right)=\omega\left(\left|\boldsymbol{e}_{i}\right|^{2}\right) / c_{s}^{2}$. Following Yuan and Schaefer [39], the pseudopotential is expressed as

$$
\psi=\sqrt{\frac{2\left(p_{\mathrm{EOS}}-\rho c_{s}^{2}\right)}{G c^{2}}},
$$

where $c=1$ is the lattice constant and $p_{\mathrm{EOS}}$ is related to the equation of state (EOS). In this work, the piecewise equation of state is adopted. For more details, interested readers are kindly directed to $[25,40]$. In addition, the following terms are added to $\bar{F}_{i}$ to describe the interaction between the 


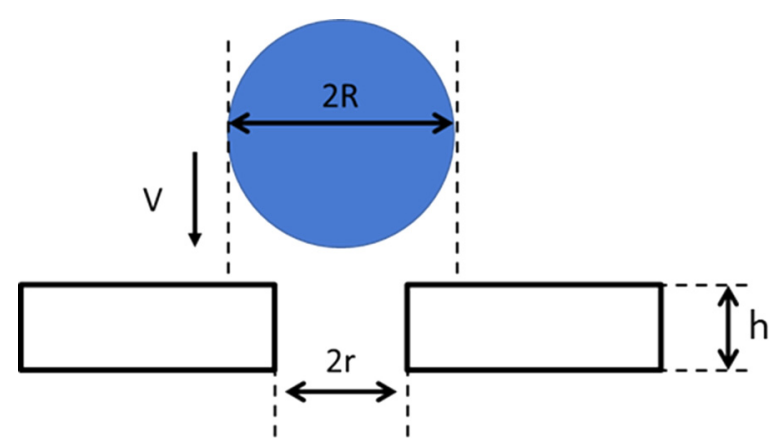

FIG. 1. Sketch of a liquid drop impacting a perforated solid surface.

fluid and a solid surface [27]:

$$
\boldsymbol{F}_{\text {ads }}=-G_{\text {ads }} \psi(\boldsymbol{x}) \sum_{i} w\left(\left|\boldsymbol{e}_{i}\right|^{2}\right) \psi(\boldsymbol{x}) s\left(\boldsymbol{x}+\boldsymbol{e}_{i} \Delta t\right) \boldsymbol{e}_{i},
$$

where $G_{\text {ads }}$ is the fluid-solid interaction strength to adjust the droplet contact angle, and $s(\boldsymbol{x})$ is an indicator function which equals 1 for solid and 0 for fluid, respectively. To achieve a large density ratio and tunable surface tension for multiphase flow in such a nonorthogonal MRT-LBM, several elements in $\tilde{\boldsymbol{F}}$ should be modified. Detailed derivations and explanations for such a modification can be seen in the study of Fei et al. [25].

\section{B. Simulation setup}

Figure 1 shows a two-dimensional sketch of the three-dimensional physical configuration in which a liquid droplet impinges on a perforated solid plate. The droplet center is aligned with the center of the pore. The radius of the liquid droplet is $R$, the thickness of the plate is $h$, the radius of the hole is $r$, and $V$ represents the impact speed.

In this study, the Weber number is defined as $\mathrm{We}=2 R \rho_{l} V^{2} / \gamma$ and the Reynolds number is $\operatorname{Re}=2 R \rho_{l} V / \eta$, where $\rho_{l}, \gamma, \eta$ are the density, surface tension, and dynamic viscosity of the droplet, respectively. The surface tension is calculated via Laplace's law $\Delta \mathrm{P}=P_{\text {int }}-P_{\text {out }}=2 \gamma / R$, where $\mathrm{P}$ is the pressure. In the following simulations, the surface tension is kept at 0.010866 in the lattice unit, which corresponds to the experimental condition. Considering that the present droplet diameter is $0.17 \mathrm{~mm}$, which is below the inertial capillary length $L_{c}=2.7 \mathrm{~mm}$ [41], the impact dynamics are mainly influenced by the capillary pressure, and the influence of gravity is negligible [41,42].

A mesh independence study is conducted by simulating a drop impacting a perforated plate with $\mathrm{We}=7.8$ and $\mathrm{Re}=39$, under different resolutions. The pore radius and the plate thickness are kept 0.4 and 0.6 times the droplet radius. While all the other parameters are fixed, the total mesh points $\left(D_{x} \times D_{y} \times D_{z}\right)$ are varied from $150 \times 150 \times 275$ to $200 \times 200 \times 367,300 \times 300 \times 550$ up to 500 $\times 500 \times 735$, respectively. $D_{x}, D_{y}$, and $D_{z}$ represent the mesh points in the $\mathrm{x}, \mathrm{y}$, and z directions, respectively. The relative errors caused by the mesh size can be written as

$$
E\left(D_{x}\right)=\frac{\left|v^{\max }\left(D_{x}\right)-v^{\max }(500)\right|}{v^{\max }(500)} \times 100 \%,
$$

where $v^{\max }\left(D_{x}\right)$ is the maximum velocity in the central line during the droplet evolution, and the following graph shows $v^{\max }$ versus the mesh resolution in $X$ direction $\left(D_{x}\right)$.

Based on Fig. 2, it is concluded that when $D_{x}$ is more than 300 , the resolution of the mesh has little influence on the simulation results. Therefore, in the following study, $D_{x}$ greater than 300 is employed. 


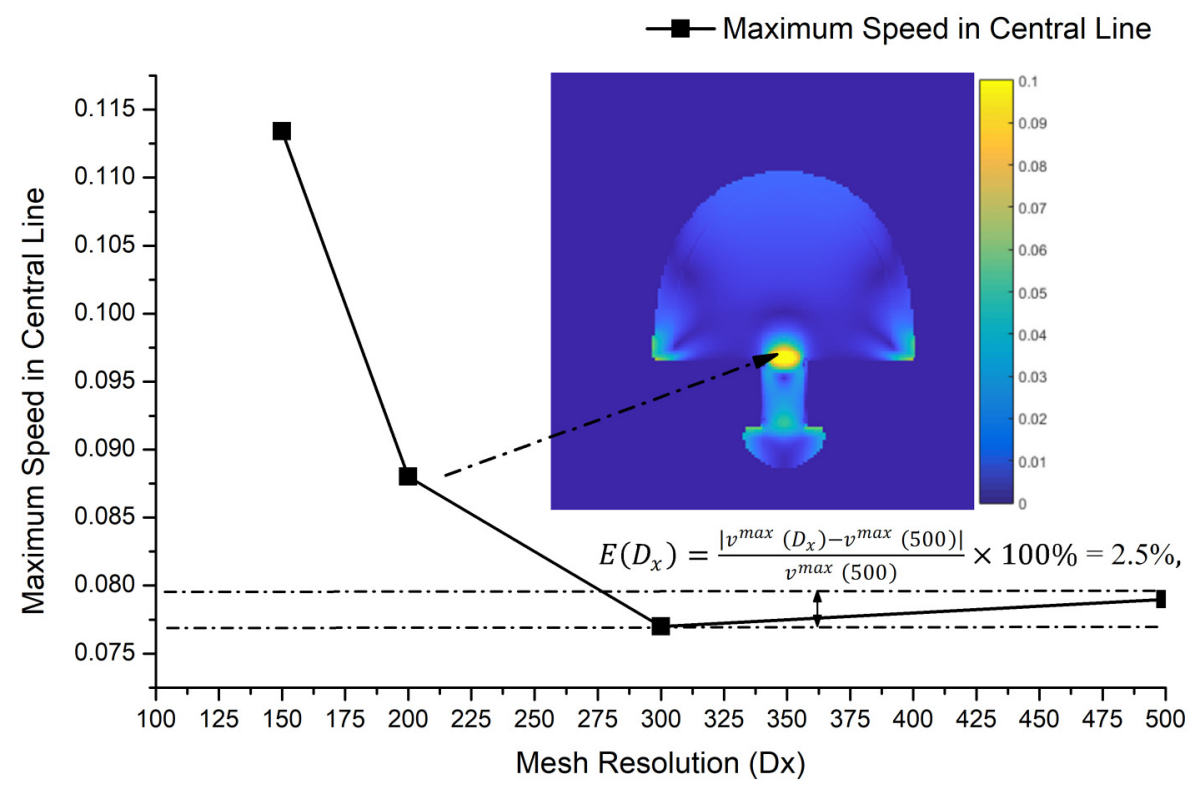

FIG. 2. Change of the maximum speed in the central line under different mesh resolutions, where the relative error between $D_{x}=300$ and $D_{x}=500$ is $2.52 \%$.

\section{Model validation}

The validation of the physical and numerical models is based on a 3.5 -mm-diameter silicone oil droplet impacting on a 0.25 -mm-thick stainless steel plate with a $450-\mu \mathrm{m}$ radius pore. The simulation results are compared with the experimental results in [17]. In the experimental study, the threshold Weber number, below which the droplet is totally captured by the plate, as a function of the Reynolds number is measured.

A series of simulations have been conducted to compare with the experimental data. The computational mesh is $400 \times 400 \times 500$, with a droplet radius of 40 in lattice units. The density ratio of the liquid and vapor phase is fixed at 800 for the silicone oil condition, and $G_{\text {ads }}$ is set as 0.035 so that the contact angle between the surface and the liquid is approximately $100^{\circ}$ [43]. The Reynolds number and Weber number are tuned by the velocity and kinematic viscosity $(v)$ of the droplet to match the experimental conditions.

As shown in Fig. 3, the simulation results reach a good agreement with the existing experimental data. Under a certain Reynolds number and above the critical Weber number, liquid is ejected from the surface and then breaks up into smaller droplets. When a Weber number lower than the critical value is used, a liquid finger transiently forms and retracts, and eventually all the liquid is captured by the surface. Besides the present results, qualitative and quantitative validation of the proposed simulation methodology has been performed in the case of droplet impact on a solid substrate [25].

The spurious currents in the pseudopotential model are regarded as an important cause for numerical instability [22]. Here we measure the spurious currents by simulating a stationary water droplet in the same simulation domain as above, while the kinematic viscosity of the droplet varies from 0.005 to 0.0125 . The average spurious velocity magnitude in the gas phase $\bar{u}_{v}$ [34] is compared with the classical single-relaxation-time (SRT) LBM, where the SRT-LBM is realized by setting all the relaxation parameters the same. Table I shows the $\bar{u}_{v}$ after 8000 iteration steps.

The average magnitude of the spurious velocities is below $10^{-3}$ when the kinetic viscosity $v$ is varied from 0.005 to 0.0125 for both methods. The nonorthogonal MRT-LBM model gives lower spurious velocity magnitudes compared with the traditional SRT-LBM model. 


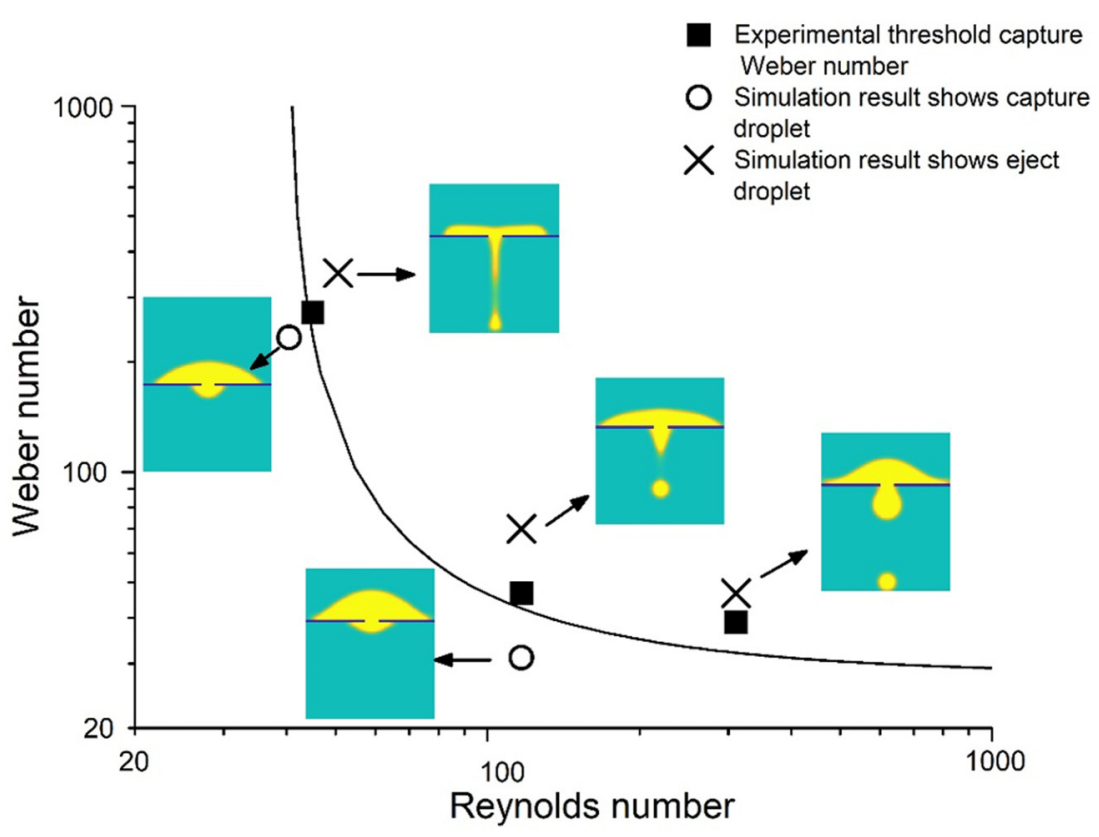

FIG. 3. Validation of the present model against experimental data [17]. Below the experimental capture Weber number, all of the droplet remains in the plate. The droplet escapes and breaks up when higher than the experimental capture Weber number is used. The solid curve $\mathrm{Re}^{*}\left(\mathrm{We}^{*}-3.6\right)=5.1 \mathrm{We}^{*}$ represents the theoretical function of the critical Weber number and the critical Reynolds number.

\section{SIMULATION RESULTS AND DISCUSSION}

In order to research the various water droplet penetration behaviors when impacting a hydrophobic surface, simulations are conducted for different Weber numbers, pore sizes, and plate thicknesses. The water droplet properties are implemented during the simulation. The density ratio and the kinematic viscosity ratio of the liquid and the vapor phases are fixed at 1000 and 20, respectively. The density is set as 0.001 for the vapor phase and the kinematic viscosity is set as 0.0075 for the liquid phase. The droplet radius is set up as 40 and the static contact angle of the solid and liquid $(\theta)$ is fixed as $123^{\circ}$ to reach the hydrophobic condition.

Two groups of simulations have been done under the plate thickness-droplet radius ratio $h / R$ of 0.4 and 0.6 , respectively. The pore size-droplet radius ratio $r / R$ is varied from 0.19 to 0.4 , and the Weber number is tuned by the impact velocity. The Ohnesorge number, $\mathrm{Oh}=\eta / \sqrt{2 \gamma \rho_{l} R}$, is kept as 0.008 according to the fixed water droplet properties. The phase diagram of the droplet states after impact is described in Figs. 4 and 5. The $x$ axis represents $r / R$ and the $y$ axis stands for the Weber number.

The phase diagram results are in good agreement with the experimental results $[17,18]$. The thicker plate will increase the viscous drag, and most of the cases show that the droplet is hanging on the plate (Fig. 6), while for the thinner plate the dynamic pressure results in the droplet

TABLE I. The average spurious velocity magnitude in the gas phase $\bar{u}_{v}$ vs liquid kinetic viscosity $\nu$.

\begin{tabular}{lcccc}
\hline \hline Method & $v=0.005$ & $v=0.0075$ & $v=0.01$ & $v=0.0125$ \\
\hline D3Q19 MRT & 0.00021 & 0.000105 & 0.000213 & 0.000285 \\
D3Q19 SRT & 0.000249 & 0.000409 & 0.000547 & 0.00063 \\
\hline \hline
\end{tabular}




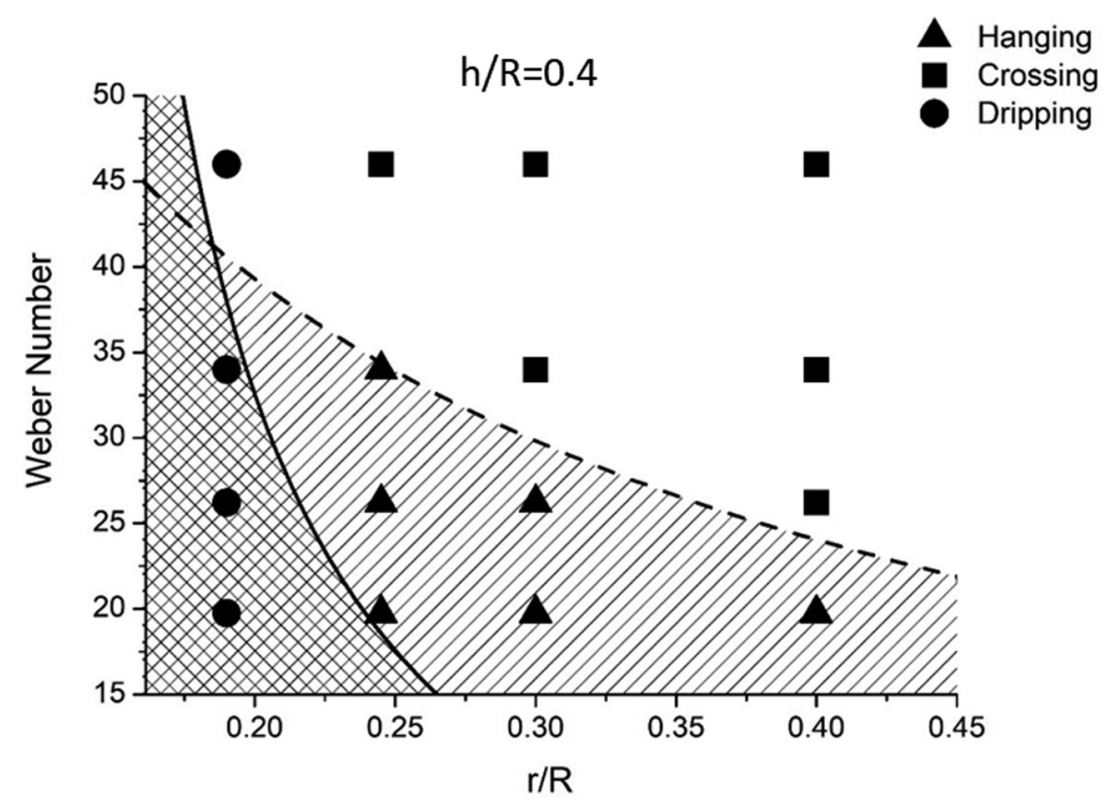

FIG. 4. The phase diagram for the $h / R=0.4$ case. The triangle indicates partial crossing of the droplet, while the major droplet is hanging on the plate. The square represents the case of the droplet totally crossing the plate. The circle describes continuously dripping liquid from the droplet. The solid line represents Eq. (15), and the dashed line stands for Eq. (17).

breaking up and totally crossing the plate (Fig. 7). What is interesting is that for both thicknesses, the droplet shows a continuously dripping state under the condition of a smaller pore size. A liquid jet forms under the plate and continuously breaks up into small droplets (Fig. 8).

The droplet impact process can be divided into the spread, rebound, permeation, and steady state. On the aspect of force balance, during the droplet evolution the dynamic pressure, viscosity, and capillary effects compete with each other. In terms of energy conversion, owing to the formation of the liquid jet and its breakup, the droplet kinetic energy converts into the surface free energy. In the following paragraph, a detailed pressure and energy analysis will be conducted.

\section{A. Pressure analysis}

For qualitative analysis, it can be observed that during the spread stage, for the hanging and totally crossing droplet cases, a liquid finger transiently forms at the bottom of the plate and breaks up (Figs. 6 and 7). The remaining droplet on the plate rebounds and permeates once again. However, for the continuously dripping cases, there is no obvious liquid finger during the spread stage (Fig. 8). Regardless of the rebound and permeation stages, a liquid finger forms at the bottom of the plate for all cases. Nevertheless, for the continuously dripping and totally crossing droplet cases, the liquid finger continuously develops and breaks up while a small liquid finger transiently occurs at the bottom of the plate and reaches equilibria for the hanging cases. Thus, the liquid finger is proved to critically affect the droplet penetration.

The formation of the liquid finger can be explained as a result of the competition between the dynamic pressure and the capillary pressure $[9,44]$. The dynamic pressure $\left(0.5 \rho V^{2}\right)$ of the droplet manages to cover the Laplace pressure on the droplet top interface [point A in Fig. 9(a), scales as $-2 \gamma / R$ ] and the Laplace pressure on the droplet bottom interface [point B in Fig. 9(a), scales as $-2 \gamma \cos \theta / r$ ], where $\theta$ is the contact angle of the liquid and solid interface [9]. Additionally, the 


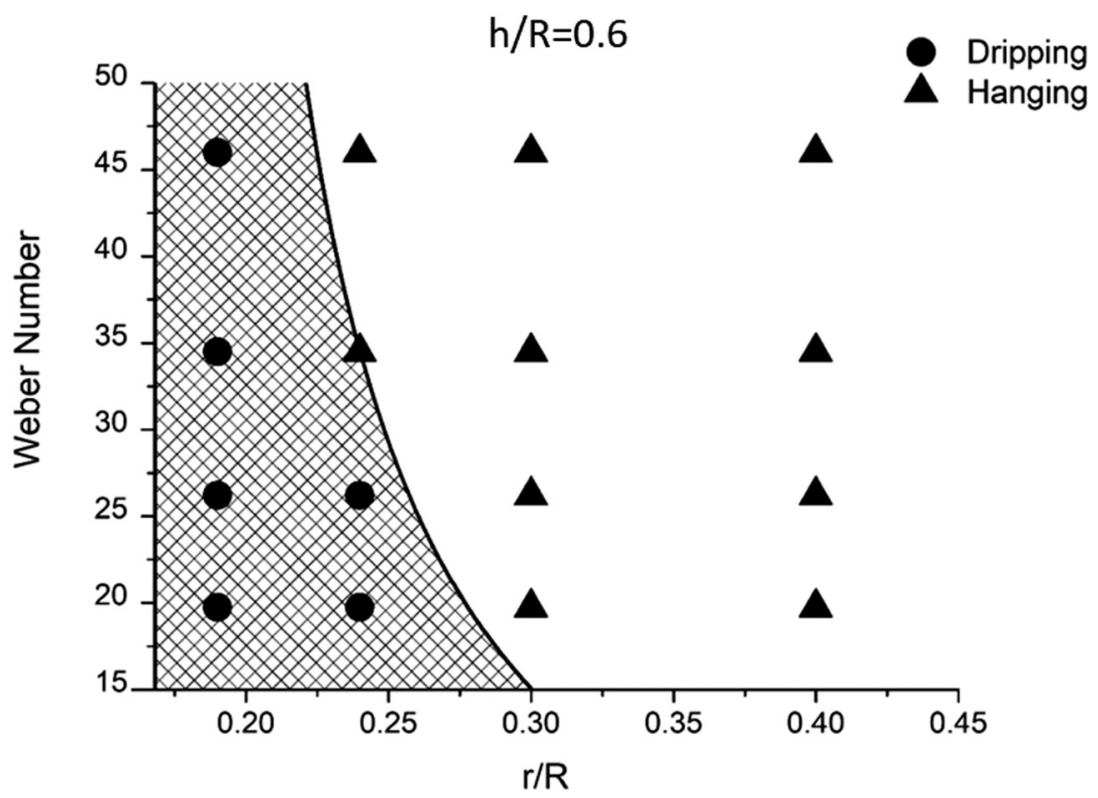

FIG. 5. The phase diagram for the $h / R=0.6$ case. The triangle indicates partial crossing of the droplet while the major droplet is hanging on the plate. The circle describes continuously dripping liquid from the droplet. The solid line represents Eq. (15).

droplet viscous losses cannot be ignored in this study owing to the large ratio between the viscous force and the capillary force, the capillary number $C a$, which is defined as $C a=\eta V R /(\gamma r) \sim$ $10[9,45]$. Considering the contribution of the plate thickness, and coupled with the viscosity dissipation function proposed in Refs. [12,46], the pressure losses caused by the viscous effect scales with $\zeta h \rho V^{2} /(2 r)$, where $\zeta$ is the viscous loss coefficient.

Since no liquid jet forms during the spread stage for the continuously dripping cases, the threshold of continuous dripping can be written as

$$
\frac{1}{2} \rho V^{2}=-\frac{2 \gamma \cos \theta}{r}-\frac{2 \gamma}{R}+\frac{\zeta h}{2 r} \rho V^{2}
$$

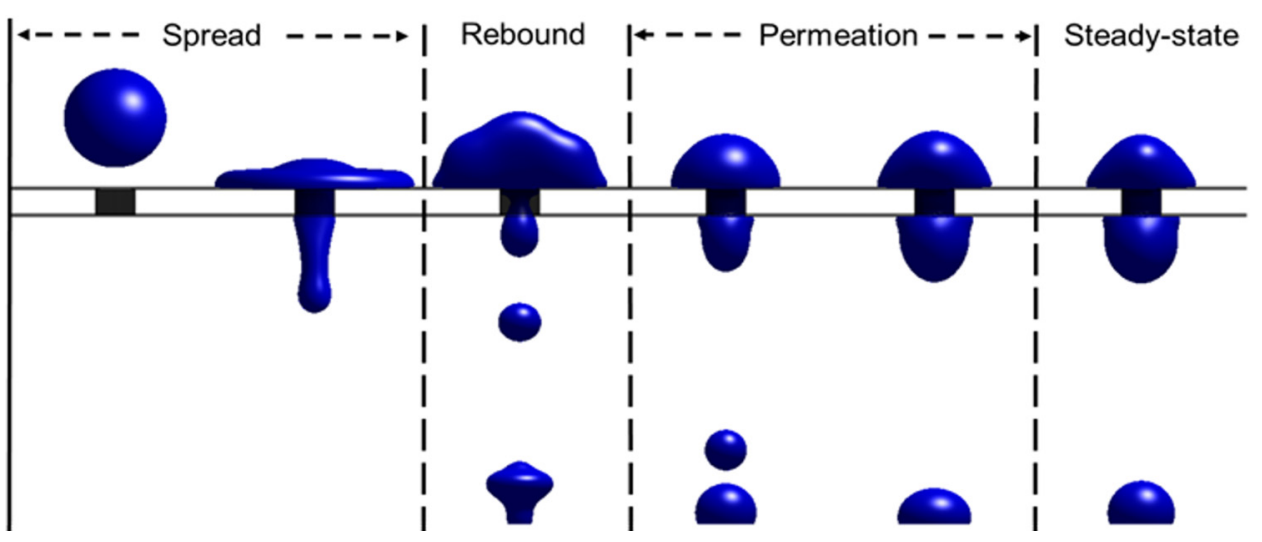

FIG. 6. The evolution of the hanging droplet under $h / R=0.6, r / R=0.4$, and We $=46$. 


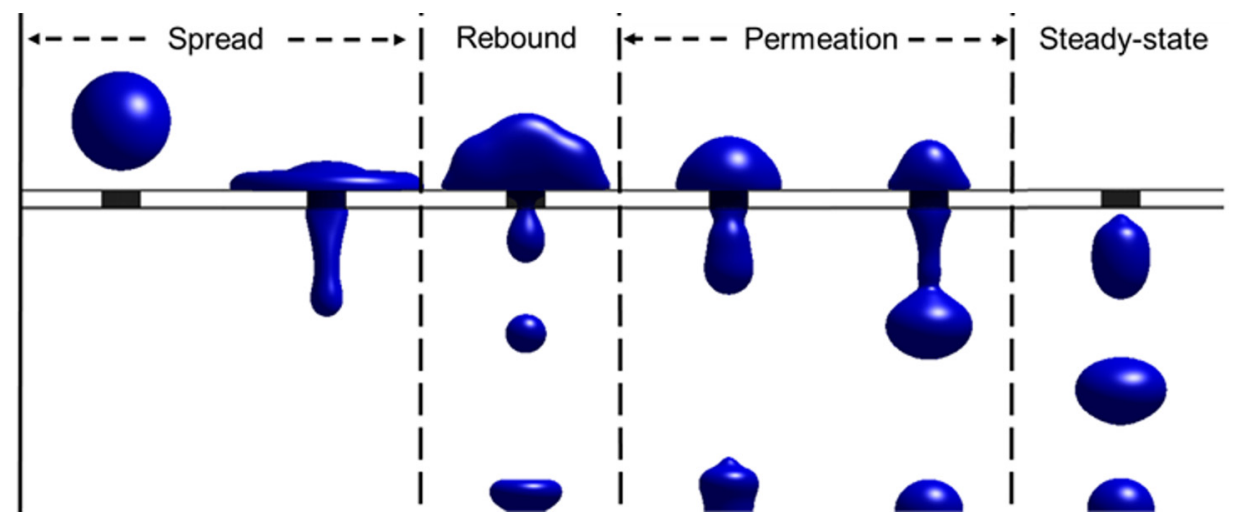

FIG. 7. The evolution of the totally crossing droplet under $h / R=0.4, r / R=0.4$, and We $=46$.

Substituting the dimensionless number We, $r / R$, and $h / R$, the above equation can be rewritten as

$$
W e=-8\left[\frac{\frac{r}{R}+\cos \theta}{\frac{r}{R}-\zeta \frac{h}{R}}\right] .
$$

For the totally crossing and hanging cases, the equilibrium of the top and bottom interface capillary forces during the rebound stage is used to identify the threshold. As shown in Fig. 9(b), the diameter of curvature of the droplet top interface can be regarded as the pore size. So the Laplace pressure of the droplet top interface approximately scales with $2 \gamma / r$. Thus

$$
\frac{1}{2} \rho V^{2}=-\frac{2 \gamma \cos \theta}{r}+\frac{2 \gamma}{r}-\frac{\zeta h}{2 r} \rho V^{2}
$$

Similarly, the above equation can be expressed as

$$
W e=8\left[\frac{1-\cos \theta}{\frac{r}{R}+\zeta \frac{h}{R}}\right],
$$

where the viscous loss coefficient $\zeta$ is chosen as 0.2705 and $\theta$ is chosen as $123.15^{\circ}$. The solid line in Figs. 4 and 5 represents Eq. (15), and the dashed line in Fig. 4 represents Eq. (17).

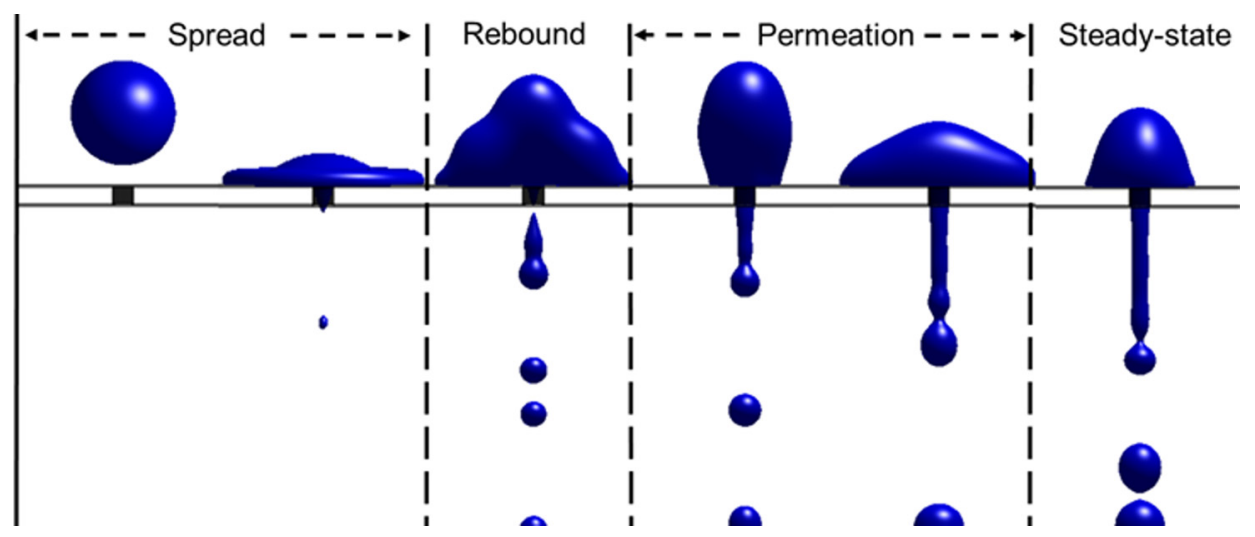

FIG. 8. The evolution of the continuously dripping droplet under $h / R=0.6, r / R=0.19$, and We $=46$. 

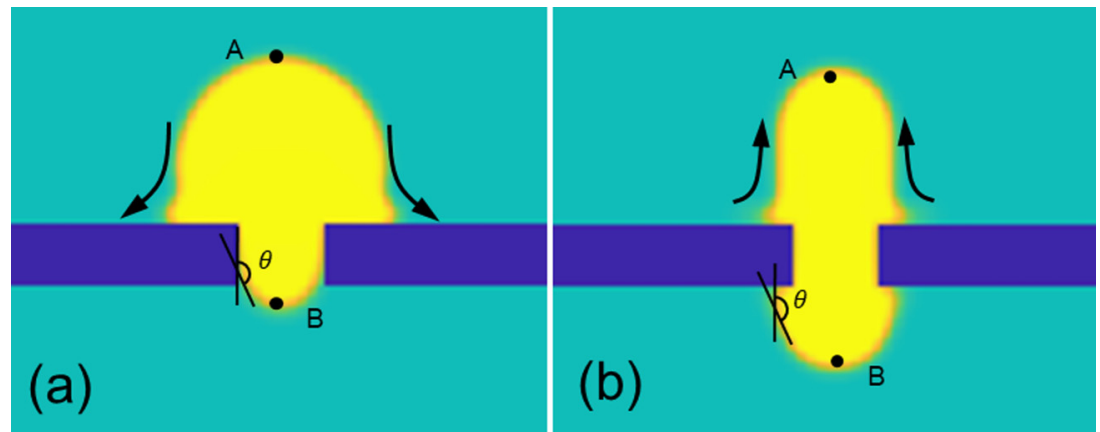

FIG. 9. The ideal model of droplet equilibrium: the left indicates the spread, and the right represents the rebound.

The present model shows good agreement with the existing simulations. It should be pointed out that an approximate calculation is used for the Laplace pressure during the rebound stage. Moreover, the influence of the viscosity term may vary in different configurations, so the accuracy of balance equations in Eqs. (15) and (17) needs to be validated over a wide range of Oh and Ca numbers.

\section{B. Energy analysis}

For more quantitative analysis, we consider the energy conversion during the droplet-plate interaction in this section. First, it is known that the kinetic energy (KE) is converted into the surface free energy (SE) during the spread stage. Furthermore, as shown in Fig. 10(a), the obvious vortex and velocity gradient occur inside the droplet and the pore. Thus viscous energy dissipation (VD) cannot be ignored [12]. The total of the kinetic energy and the surface energy can be written as [47]

$$
\mathrm{TE}=\mathrm{KE}+\mathrm{SE},
$$

where TE is the total useful energy, KE is computed as $\iiint 0.5 \rho v^{2} d \Omega$, and SE is $\gamma S . \Omega$ is the droplet volume and $S$ is the liquid interface area. The viscous energy dissipation can be written as [47]:

$$
\mathrm{VD}=\int_{0}^{t} \int_{0}^{\Omega} \phi d \Omega \mathrm{d} t
$$
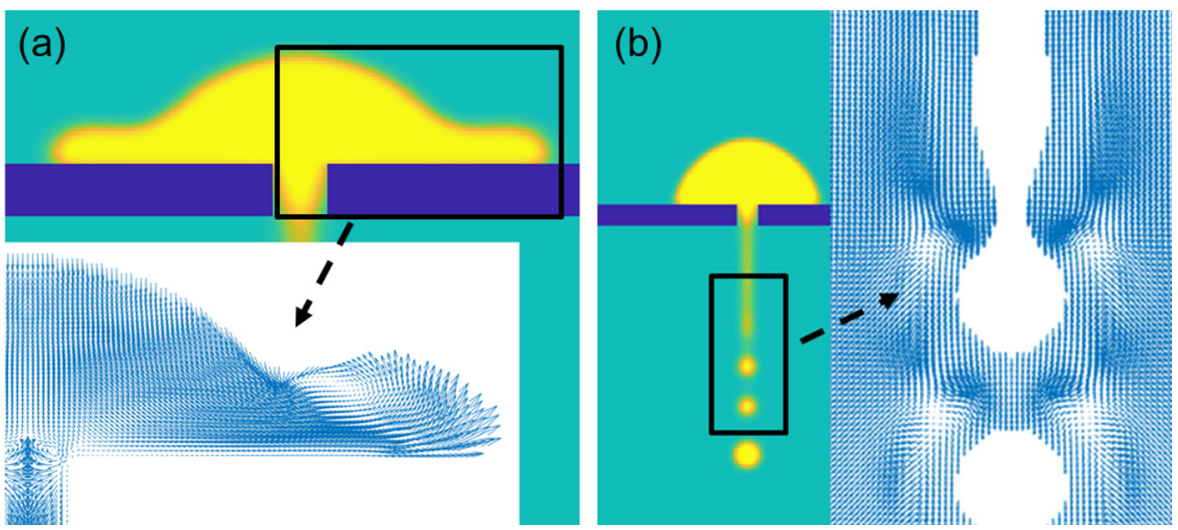

FIG. 10. The velocity vector field during the evolution. The left figure indicates the velocity vector field inside the droplet in the spread stage. The right figure represents the velocity vector field outside the droplet in the permeation stage. 


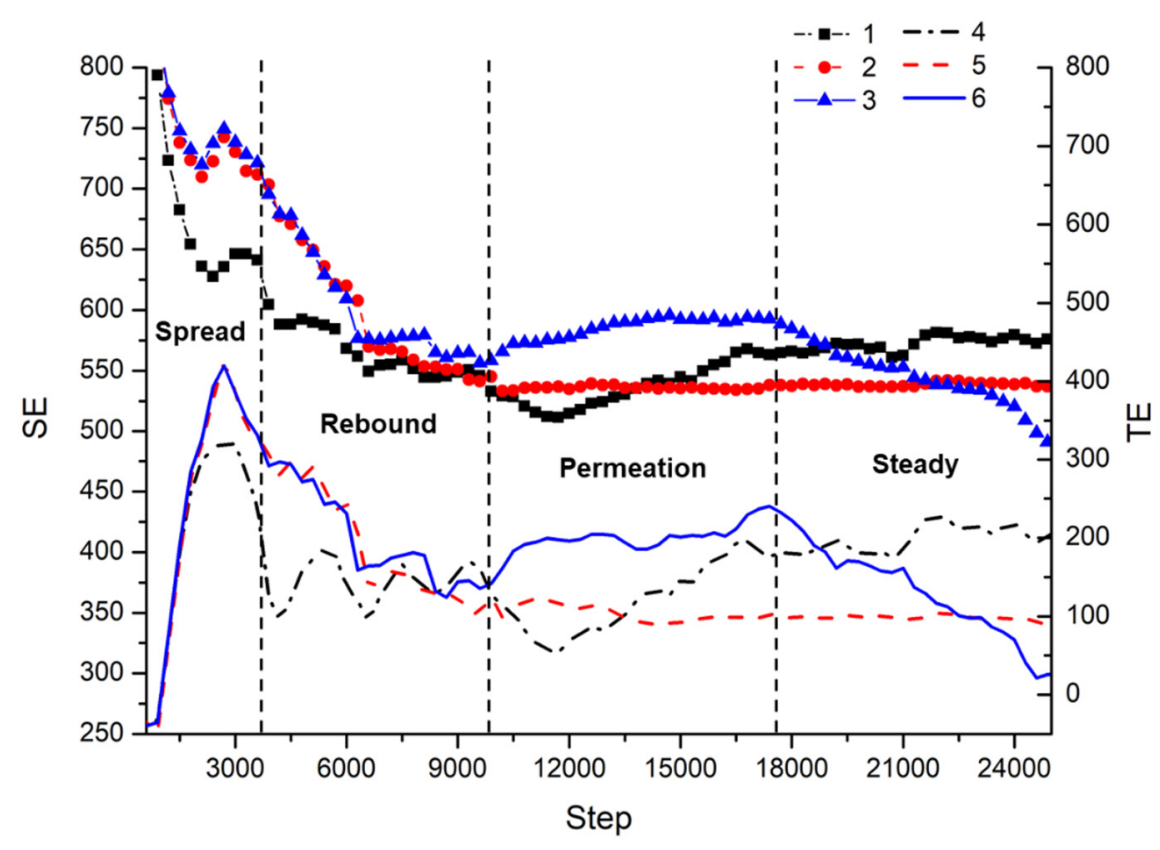

FIG. 11. The surface energy (left $Y$-axis, represented by line) and total useful energy (right $Y$-axis, represented by line and symbol) evolution of the droplet. The total useful energy: 1 . the continuously dripping case, 2 . the hanging case, 3 . totally crossing case; The surface energy: 4 . the continuously dripping case, 5 . the hanging case, 6 . the totally crossing case.

where $t$ is the time, $\int_{0}^{\Omega} \phi d \Omega$ represents the droplet viscous dissipation rate (VDR), and [48]

$$
\phi=\eta\left[2\left(\frac{\partial u}{\partial x}\right)^{2}+2\left(\frac{\partial v}{\partial y}\right)^{2}+2\left(\frac{\partial w}{\partial z}\right)^{2}+\left(\frac{\partial u}{\partial y}+\frac{\partial v}{\partial x}\right)^{2}+\left(\frac{\partial v}{\partial z}+\frac{\partial w}{\partial y}\right)^{2}+\left(\frac{\partial w}{\partial x}+\frac{\partial u}{\partial z}\right)^{2}\right] .
$$

The following three cases, hanging (We $=46, r / R=0.4, h / R=0.6$ ), total crossing (We $=46$, $r / R=0.4, h / R=0.4$ ), and continuous dripping (We $=46, r / R=0.19, h / R=0.4$ ), are taken as examples for the quantitative analysis of the energy evolution. As shown in Fig. 11, during the spread stage the conversion between the surface energy and the kinetic energy for the hanging and totally crossing cases is similar. However, during the rebound and permeation stages, the thicker plate will lead to a higher viscous dissipation rate (see Fig. 12). As a result, the viscous energy loss increases, which leads to the hanging droplet.

What also needs to be mentioned is that due to the formation and breakup of the liquid jet during the spread and rebound stage, the surface free energy and kinetic energy for the hanging and totally crossing cases are obviously higher than the continuously dripping cases. As shown in Eq. (20), the viscous dissipation rate is determined by the velocity gradient, and thus a lower viscous dissipation rate is seen for the continuously dripping case (shown in Fig. 12), which in turn results in the higher total useful energy for the continuously dripping case in the late stage (shown in Fig. 11). The development of the liquid finger is caused by the higher total useful energy during the permeation stage, which can be observed in the totally crossing and continuously dripping droplet cases (see Figs. 7 and 8).

Considering the higher total useful energy for the totally crossing and continuously dripping cases during the permeation stage, the liquid jet continuously develops and then breaks up. As 


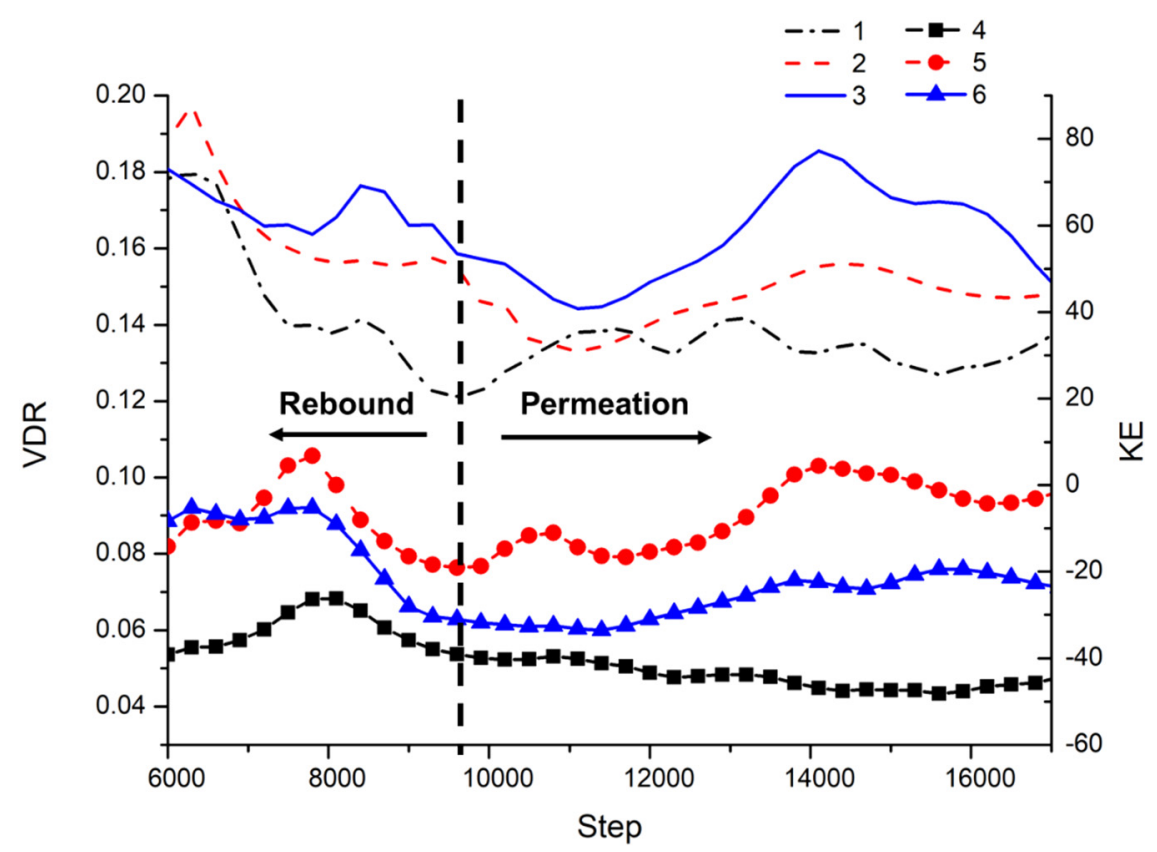

FIG. 12. The kinetic energy (right $y$ axis, represented by line) and viscous dissipation rate (left $y$ axis, represented by line and symbol) for the rebound and permeation stages. The kinetic energy: 1 . the continuously dripping case, 2. the hanging case, and 3. the totally crossing case. The viscous dissipation rate: 4. the continuously dripping case, 5 . the hanging case, and 6 . the totally crossing case.

shown in Fig. 10(b), the capillary instability caused by viscous drag of the ambient fluid can be observed. According to the theory of capillary instability, the jet breaks up into small droplets when the wavelength of the liquid jet is greater than the critical wavelength. Meanwhile, the size of the droplets after the jet breakup will be proportional to the liquid jet diameter [10,49]. Thus, the bigger liquid cylinder breaks up into larger droplets, leading to the remaining droplet crossing. The smaller liquid column breaks up into small droplets, resulting in a continuously dripping state.

\section{CONCLUSION}

This study uses a nonorthogonal multiple-relaxation-time LBM to numerically simulate a water droplet impacting a hydrophobic plate with a cylindrical pore. The LBM formulation is shown to correctly capture the dynamic interaction between the liquid droplet and the perforated solid surface, in comparison with existing experimental results. Three distinctive regimes of droplet dynamics: hanging, totally crossing and continuously dripping, have been observed for a wide range of impact speed, pore size, and plate thickness. As a result, phase diagrams have been constructed to describe the final states of the droplet. Based on a qualitative analysis of the droplet evolution process, the liquid finger at the bottom of the plate has been shown to significantly affect the droplet impact outcome.

Based on the balance among the droplet dynamic pressure, capillary pressure, and the viscous pressure losses during the spread and rebound stages, the criteria determining the final states of the impacting droplet have been proposed [Eqs. (15) and (17)]. These theoretical criteria show excellent agreement with the numerical simulation results. Based on energy analysis, the viscous energy dissipation leads to the reduction of the total useful energy, which results in the hanging drop state. The higher total useful energy contributes to the formation and development of the liquid jet. Driven by the capillary instability, the continuously developing liquid jet breaks up into small 
droplets, which finally results in the totally crossing and continuously dripping states. It is noted that the present work is focused on the high-Ca region, in which the proposed criteria [Eqs. (15) and (17)] for different final states are well verified. In the future it would be informative to study the validity or otherwise of such criteria in a much wider range of hydrodynamic parameters and surface wettability. It would also be very interesting to investigate how the impacting dynamics would change if the center of the droplet is not aligned with that of the pore center.

\section{ACKNOWLEDGMENTS}

This work was supported by the UK Engineering and Physical Sciences Research Council (EPSRC) under the project "UK Consortium on Mesoscale Engineering Sciences (UKCOMES)" (Grant No. EP/R029598/1) and the project "'HiLeMMS': High-Level Mesoscale Modelling System" (Grant No. EP/P022243/1).

[1] T. Kamal, R. Watkins, Z. Cen, J. Rubinstein, G. Kong, and W. M. Lee, Design and fabrication of a passive droplet dispenser for portable high resolution imaging system, Sci. Rep. 7, 41482 (2017).

[2] L. E. Murr and W. L Johnson, 3D metal droplet printing development and advanced materials additive manufacturing, J. Mater. Res. Technol. 6, 77 (2017).

[3] A. L. Yarin, Drop impact dynamics: Splashing, spreading, receding, bouncing ..., Annu. Rev. Fluid Mech. 38, 159 (2006).

[4] A. Y. Hsiao, Y. C. Tung, C. H. Kuo, B. Mosadegh, R. Bedenis, K. J. Pienta, and S. Takayama, Micro-ring structures stabilize microdroplets to enable long term spheroid culture in 384 hanging drop array plates, Biomed. Microdevices 14, 313 (2012).

[5] S. Y. Teh, R. Lin, L. H. Hung, and A. P. Lee, Droplet microfluidics, Lab Chip 8, 198 (2008).

[6] P. Zhu and L. Wang, Passive and active droplet generation with microfluidics: A review, Lab Chip 17, 34 (2017).

[7] T. Han, H. Noh, H. S. Park, and M. H. Kim, Effects of wettability on droplet movement in a V-shaped groove, Sci. Rep. 8, 16013 (2018).

[8] A. H. Lefebvre and V. G McDonell, Atomization and Sprays (CRC Press, Boca Raton, FL, 2017).

[9] A. Delbos, E. Lorenceau, and O. Pitois, Forced impregnation of a capillary tube with drop impact, J. Colloid Interface Sci. 341, 171 (2010).

[10] S. P. Lin and R. D. Reitz, Drop and spray formation from a liquid jet, Annu. Rev. Fluid Mech. 30, 85 (1998).

[11] L. Rayleigh, On the instability of jets, Proc. London Math. Soc. s1-10, 4 (1878).

[12] M. Pasandideh-Fard, Y. M. Qiao, S. Chandra, and J. Mostaghimi, Capillary effects during droplet impact on a solid surface, Phys. Fluids 8, 650 (1996).

[13] G. S. Watson, M. Gellender, and J. A. Watson, Self-propulsion of dew drops on lotus leaves: A potential mechanism for self cleaning, Biofouling 30, 427 (2014).

[14] N. F. Himma, N. Prasetya, S. Anisah, and I. G. Wenten, Superhydrophobic membrane: Progress in preparation and its separation properties, Rev. Chem. Eng. 35, 211 (2019).

[15] A. Kumar, A. Tripathy, Y. Nam, C. Lee, and P. Sen, Effect of geometrical parameters on rebound of impacting droplets on leaky superhydrophobic meshes, Soft Matter 14, 1571 (2018).

[16] G. Zhang, M. A. Quetzeri-Santiago, C. A. Stone, L. Botto, and J. R. Castrejón-Pita, Droplet impact dynamics on textiles, Soft Matter 14, 8182 (2018).

[17] É. Lorenceau and D. Quéré, Drops impacting a sieve, J. Colloid Interface Sci. 263, 244 (2003).

[18] S. A. Kooij, A. M. Moqaddam, T. C. de Goede, D. Derome, J. Carmeliet, N. Shahidzadeh, and D. Bonn, Sprays from droplets impacting a mesh, J. Fluid Mech. 871, 489 (2019).

[19] R. Haghani, M. H. Rahimian, and M. Taghilou, LBM simulation of a droplet dripping down a hole, Eng. Appl. Comput. Fluid Mech. 7, 461 (2013). 
[20] M. Sussman, P. Smereka, and S. Osher, A level set approach for computing solutions to incompressible two-phase flow, J. Comput. Phys. 114, 146 (1994).

[21] Q. Li, K. H. Luo, Q. J. Kang, Y. L. He, Q. Chen, and Q. Liu, Lattice Boltzmann methods for multiphase flow and phase-change heat transfer, Prog. Energy Combust. Sci. 52, 62 (2016).

[22] L. Chen, Q. Kang, Y. Mu, Y. L. He, and W. Q. Tao, A critical review of the pseudopotential multiphase lattice Boltzmann model: Methods and applications, Int. J. Heat Mass Transfer 76, 210 (2014).

[23] Y. Wei, H. Yang, H. S. Dou, Z. Lin, Z. Wang, and Y. Qian, A novel two-dimensional coupled lattice Boltzmann model for thermal incompressible flows, Appl. Math. Comput. 339, 556 (2018).

[24] S. Mukherjee, A. Zarghami, C. Haringa, K. van As, S. Kenjereš, and H. E. Van den Akker, Simulating liquid droplets: A quantitative assessment of lattice Boltzmann and volume of fluid methods, Int. J. Heat Fluid Flow 70, 59 (2018).

[25] L. Fei, J. Du, K. H. Luo, S. Succi, M. Lauricella, A. Montessori, and Q. Wang, Modeling realistic multiphase flows using a non-orthogonal multiple-relaxation-time lattice Boltzmann method, Phys. Fluids 31, 042105 (2019).

[26] Q. Li, K. H. Luo, and X. J. Li, Lattice Boltzmann modeling of multiphase flows at large density ratio with an improved pseudopotential model, Phys. Rev. E 87, 053301 (2013).

[27] Q. Li and K. H. Luo, Achieving tunable surface tension in the pseudopotential lattice Boltzmann modeling of multiphase flows, Phys. Rev. E 88, 053307 (2013).

[28] D. A. Hoang, L. M. Portela, C. R. Kleijn, M. T. Kreutzer, and V. Van Steijn, Dynamics of droplet breakup in a T-junction, J. Fluid Mech. 717, R4 (2013).

[29] Y. Chen and Z. Deng, Hydrodynamics of a droplet passing through a microfluidic t-junction, J. Fluid Mech. 819, 401 (2017).

[30] Z. Guo, C. Zheng, and B. Shi, Discrete lattice effects on the forcing term in the lattice Boltzmann method, Phys. Rev. E 65, 046308 (2002).

[31] Q. Liu, Y. L. He, D. Li, and Q. Li, Non-orthogonal multiple-relaxation-time lattice Boltzmann method for incompressible thermal flows, Int. J. Heat Mass Transfer 102, 1334 (2016).

[32] P. Lallemand and L. S. Luo, Theory of the lattice Boltzmann method: Dispersion, dissipation, isotropy, Galilean invariance, and stability, Phys. Rev. E 61, 6546 (2000).

[33] D. d'Humieres, Multiple-relaxation-time lattice Boltzmann models in three dimensions, Philos. Trans. R. Soc. London, Ser. A 360, 437 (2002).

[34] D. Lycett-Brown and K. H. Luo, Multiphase cascaded lattice Boltzmann method, Comput. Math. Appl. 67, 350 (2014).

[35] Q. Li, D. H. Du, L. L. Fei, and K. H. Luo, Three-dimensional non-orthogonal MRT pseudopotential lattice Boltzmann model for multiphase flows, Comput. Fluids 186, 128 (2019).

[36] L. Fei and K. H. Luo, Consistent forcing scheme in the cascaded lattice Boltzmann method, Phys. Rev. E 96, 053307 (2017).

[37] L. Fei, K. H. Luo, and Q. Li, Three-dimensional cascaded lattice Boltzmann method: Improved implementation and consistent forcing scheme, Phys. Rev. E 97, 053309 (2018).

[38] X. Shan and H. Chen, Lattice Boltzmann model for simulating flows with multiple phases and components, Phys. Rev. E 47, 1815 (1993).

[39] P. Yuan and L. Schaefer, Equations of state in a lattice Boltzmann model, Phys. Fluids 18, 042101 (2006).

[40] C. E. Colosqui, G. Falcucci, S. Ubertini, and S. Succi, Mesoscopic simulation of non-ideal fluids with self-tuning of the equation of state, Soft Matter 8, 3798 (2012).

[41] B. E. Rapp, Microfluidics: Modeling, Mechanics and Mathematics (William Andrew Publishing, Norwich, NY, 2016).

[42] A. Bedarkar, X. F. Wu, and A. Vaynberg, Wetting of liquid droplets on two parallel filaments, Appl. Surf. Sci. 256, 7260 (2010).

[43] Q. Li, K. H. Luo, Q. J. Kang, and Q. Chen, Contact angles in the pseudopotential lattice Boltzmann modeling of wetting, Phys. Rev. E 90, 053301 (2014).

[44] D. Soto, H. L. Girard, A. Le Helloco, T. Binder, D. Quéré, and K. K. Varanasi, Droplet fragmentation using a mesh, Phys. Rev. Fluids 3, 083602 (2018). 
[45] D. Or, Scaling of capillary, gravity and viscous forces affecting flow morphology in unsaturated porous media, Adv. Water Resour. 31, 1129 (2008).

[46] S. Chandra and C. T. Avedisian, On the collision of a droplet with a solid surface, Proc. R. Soc. London, Ser. A 432, 13 (1991).

[47] G. Finotello, J. T. Padding, N. G. Deen, A. Jongsma, F. Innings, and J. A. M. Kuipers, Effect of viscosity on droplet-droplet collisional interaction, Phys. Fluids 29, 067102 (2017).

[48] C. He, X. Xia, and P. Zhang, Non-monotonic viscous dissipation of bouncing droplets undergoing offcenter collision, Phys. Fluids 31, 052004 (2019).

[49] H. Teng, C. M. Kinoshita, and S. M. Masutani, Prediction of droplet size from the breakup of cylindrical liquid jets, Int. J. Multiphase Flow 21, 129 (1995). 